\title{
COVID- 19 and Female Health-Care Workers: An Exploration of Challenges
}

\section{Shazaf Masood Sidhu}

The Peace Gong Global Coordinator, Aga Khan University Hospital, Karachi, Pakistan

Corresponding author: shazafmasood@gmail.com

Received: 17 Sept., 2020

Revised: 29 Nov., 2020

Accepted: 04 Dec., 2020

\begin{abstract}
While the COVID-19 pandemic continues to surge unabated across the world, an important dimension to the entire health-care architecture is the challenge faced by female health-care workers. The risks and rigors involved in the care of Covid-19 patients are enormous both for female and male health-care workers and professionals. However, female health-care workers face more complex challenges like having to deal with work-life balance, inadequate representation in senior policy planning structures of health-care institutions and in many cases being at a disadvantageous position in situations of unpaid leave. This paper through in-depth interviews of female health-care workers will try to explore the multiple challenges they face and what can be done to ensure gender equity in the health-care architecture.
\end{abstract}

Keywords: female health-care workers, gender equity, work-life balance

Notwithstanding systemic issues that foster unhealthy work environments, with expectations of 24/7 availability and a persistent life-work imbalance which many female health-care workers face, the COVID-19 pandemic has brought in more complex challenges to these workers. Whether doctors, nurses or other women in the health care system, all are facing unique issues in the face of the tsunami of COVID-19 across the world.

Across the world the tsunami of COVID-19 is playing havoc- destroying lives and livelihood. While large number of people are dying, a greater number of people are losing their livelihood due to continued lockdowns and strict restrictions across nations. Ever since the advent of the dreaded pandemic, the health care systems in all communities remain blindsided on how to deal with it, the correct approaches to treat patients and the medicines to be given. Majority of the COVID patients who require hospitalization require continuous critical care. With a large number of patients having to be given ICU and ventilator support, the challenges before healthcare workers have become manifold- right from patient management to counselling their family members. While the health-care workers- whether female or male face similar kinds of uncertain and trying situations, the challenges before female workers are more diverse and complex.

This paper will try to explore the complex challenges which female health care workers are facing. Through in-depth interviews of 25 female health workers and the writers own experience, the paper will try to build on how female health care workers are trying to overcome the pandemic which is affecting all aspects of lives and almost everyone around the globe. Convenience sampling technique

How to cite this article: Sidhu, S.M. (2020). COVID- 19 and Female Health-Care Workers: An Exploration of Challenges. Int. J. Peace, Edu. Dev., 8(02): 55-58.

Source of Support: None; Conflict of Interest: None 
was used to identify the female health-workers for the in-depth interviews and all of them are part of the writer's circle of doctors and health-care workers like nurses.

\section{Overcoming the complex challenges of female health-care workers during the pandemic}

Boniol et al. (2019) points out to an analysis of 104 countries conducted by the World Health Organization which points out that approximately 70 per cent of the global health-care workforce is made up of women.

In this backdrop, if we look at the percentage of female health-care workers who were infected, we find that in Italy, Spain, and USA (69 per cent, 75.5 per cent, 73 per cent respectively) were infected. (UN Women, 2020).

Talking about the unique challenges during the era of Covid-19, Dr Fatima Mir, Infectious Diseases Department, Aga Khan University Hospital, Karachi notes how the work-life has changed for health-care provider across the world. She points out that those involved in Covid-19 critical care treatment have to face testing times by wearing protective equipment for the entire shift which entails high physical demand. For female health-care workers it is a big challenge but there is no way out.

Mir's views can be accentuated by the writer's own experience of attending to patients in Covid wards. Many a times one has to wear PPE kits straight for 15-16 hours at a stretch and there is always anxiety if one's masks or googles have fitted properly. There are inherent risks of catching the infection or taking the virus home. Many female health-care workers were forced to shift to solitary accommodations while many others were quarantined.

This struggle has been highlighted by female doctors from other countries too. Upadhyay (2021) talking to female doctors as part of International Women's Day shares the views of Dr Surabhi Siddhartha, Consultant, Obstetrician \& Gynaecologist, Motherhood Hospital, Kharghar. She explains, "Long working hours made life miserable. "Depression, anxiety, paranoia, and nervous breakdown were common during the year. It was like we were fighting an unknown entity with no concrete treatment. The doctors around the world were learning regarding the virus as it was unknown. We were grueling inside our PPEs and eating, drinking, or even accessing the washroom was a task. There were days when I would blackout owing to the continuous inhaling of my own $\mathrm{CO}_{2}$ in the PPE. But our only focus was to provide uninterrupted medical care to the patient."

The author interviewed Iqra, a seven-month pregnant nurse in the Covid ward of Aga Khan University Hospital, Karachi. She talks about another dimension to the challenges faced by women health-care workers. The pandemic has rendered her as a single working parent as her husband's business has come to a standstill. Talking of her dual responsibilities, she says, "It is not easy, but I believe I'm a lot stronger today for we are two (referring to her baby). I'm a single earning parent at this point; my husband is a businessman but due to COVID-19 a lot has been changed and he is going through major financial crises. But even in this tough time what kept me going are these responsibilities- my responsibility towards my family and my profession. My patients need me the most at this point and I can't be selfish and step back."

In fact, all the female health-care workers who were interviewed for this paper talked at length of the tough dual responsibilities- exacting and risky duties as a health worker and family responsibilities. The female health-care workers pointed out on how as a group, women physicians have to spend proportionately more time on home and family care activities than their male counterparts. In this context, Brubaker (2020) talks about how the COVID-19 pandemic has disrupted common activities, such as meal planning and preparation, family and social activities, exercise or sport, spiritual practices, shopping, and leisure. "Many of these changes disproportionately affect women, who often are leading efforts to find an acceptable new normal," she adds.

Dr Asha Mahesh, a gynecologist and obstetrician 
points out to a critical challenge facing female physician due to rigorous schedule- the problem of burnout. She argues that at no point of time a doctor can compromise their role as critical care physician and are duty bound to provide the highest level of care to the patients. Besides, they are challenged with their role as either as a mother, wife or a daughter. Hence female hospitalists are always at risk for increased burnout, she argues.

The doctors who were interviewed talked at length on the risks and consequences of burnout. They felt that early burnout could lead to increase in negative emotions, stress and tension, blackouts, possibilities of poor performance, depression, health care workers actually being forced to leave the profession and, in many cases, unfortunately leading to suicide. The doctors felt that burnout could be aggravated by issues of gender inequities at workplace, possibilities of biasness whether real or perceived in issues like promotion or compensation and household factors like having to also look after children at home.

An important insight which Dr Rufina Ali pointed out in the context of female health-care workers were the difficulties faced by them in case of being infected. For male health-care workers, it is much easier to self-isolate in homes. But as females have to take care of their children and family at large, it is much more challenging on how to remain apart from their children.

The in-depth interviews revealed another important aspect of managing the pandemic. The female health-care workers felt that a dynamic and alert management to handle the pandemic was needed along with an empathetic and strategic leadership. The drawback these health-care workers felt was that there was little representation of women at the top leadership positions whether at the level of hospitals or policy making. This leadership gap leads to underrepresentation of issues relating to women health-care workers, they felt. For instance, Dr Ayisha Afzal Qureshi points out that there could be large number female health-care workers at lower level but due to poor opportunities, very few are able to reach the leadership positions. The policies that are framed in the context of hospitals hence remain gender-biased, she points out.

In this backdrop, Jones et al. (2020) arguing on the problems that arise due to gender inequalities in the leadership position of hospital management points out, "For example leaders of a hospital medicine group may create mandatory 'backup coverage' for night and weekend shifts for their group during surge periods of the pandemic without considering implications for childcare."

Another important challenge in the Covid-era encapsulated by Dr Aiman Raees, department of gastroenterology was on how to communicate with patients or their relatives in emotionally charged situations when people are suffering and dying alone. However, in this case studies point out on gender-related behavioral differences in communication to patients among physicians. Jefferson et al. (2013) notes how females engaged in a more empathetic manner with their patients compared to their male colleagues.

The in-depth interviews in this context revealed that female health-care workers were more equipped to handle emotions that their male counterparts. With deaths and critical conditions being quite frequently encountered vis-à-vis Covid patients, female healthcare workers, even though they are overworked tend to reach out to the affected families with empathy and compassion, the respondents felt. "I feel it is our duty to try and provide emotionally support as much as possible to the family of a critical care patient," Dr Zainab Zaidi notes in this context. Talking about her own experience, she pointed out that due to the constant barrage of messages in the media and knowledge about the condition of many patients, a very large number of families of Covid patients start believing that is all over as we start treatment in the hospitals. The doctors observed that a feeling of gloom and desperation along with depression dawns in the families. While trying not to give false assurances, it is extremely critical to help and encourage them that all was not lost. We doctors need to learn the art of positive messaging and help the families keep patience, the doctors opined. 
The in-depth interviews revealed another important dimension which is related to pay gaps based on gender inequalities. Many frontline health care workers work on contract or daily wage basis. In situations of health crisis at home, many of them have to go for unpaid leave. The female health-care workers felt that in events of health crisis involving a family member at home, it is the female member who invariably has to take charge. Those working on contract or as daily wage workers have to face the brunt, they added.

\section{CONCLUSION}

The in-depth interviews of female health-care workers offered different perspectives on the challenges faced by them in comparison to male health-care workers. Some of the important recommendations which came up during these interviews included:

$\odot$ Need for further research on direct and indirect effects of Covid-19 on female health- care workers: Those interviewed unanimously felt that more in-depth research was needed in this regard as it will help in policy formulation and execution.

$\odot$ Need for serious efforts by the top management in different health -care institutions to ensure proper representation of women in senior positions.

$\odot$ Recognition of women health-care workers: While appreciating the role of all in the war against Covid-19, the respondents felt that since women health-care workers faced multiple challenges, there should be a mechanism for recognition of their efforts. $\odot$ Development of a mechanism for fair compensation: As the respondents felt that many daily wage or contractual female health-care workers were many times at disadvantageous position compared to their male counterparts, it would be prudent on the management of the health-care institutions to devise a policy for fair compensation.

\section{REFERENCES}

Boniol,M., McIsaac, M., Xu, L., Wuliji, T., Diallo, K. and Campbell, J. 2019. Geneva: World Health Organization; Gender equity in the health workforce: analysis of 104 countries; Working paper https://apps.who.int/iris/bitstream/ handle/10665/311314/WHO-HIS-HWF-Gender-WP12019.1-eng.pdf?sequence=1\&isAllowed=y Date: March, 2020; Date accessed: November 22, 2020.

Covid-19 emerging gender data and why it matters; https:// data.unwomen.org/resources/covid-19-emerginggender-data-and-why-it-matters; Date: June 26, 2020. Date accessed: October 30, 2020.

Jefferson, L., Bloor, K., Birks, Y., Hewitt, C. and Bland, M. 2013. Effect of physicians' gender on communication and consultation length: A systematic review and metaanalysis. J. Health Serv. Res. Policy, 18: 242-248.

Jones, Y., Durand, V., Morton, K., Ottolini, Mary; Shaughnessy, E., Spector, N. and O'Toole, Jennifer. 2020. Collateral Damage: How COVID-19 Is Adversely Impacting Women Physicians; J. Hosp. Med., 15(8): 507-509.

Upadhyay, P. 2021. International Women's Day: Female doctors share their challenges and aspirations; https://www.indiatoday.in/ india/story/international-women-s-day-female-doctors-sharetheir-challenges-and-aspirations-1776721-2021-03-08. 